\title{
Effect of Home Visit Oral Health Education on Xerostomia in Older Adults
}

\author{
Ji-Yun $\mathrm{Ki}^{1}$, Jong-Hwa Jang ${ }^{1,2}$ \\ ${ }^{1}$ Department of Public Health Science, Graduate School of Natural Science, Dankook University, Korea \\ ${ }^{2}$ Department of Dental Hygiene, College of Health Science, Dankook University, Korea
}

Objectives: In the approaching super-aged society, the oral health problem of the elderly is becoming more important. A major oral problem in the elderly is dry mouth, which causes various oral diseases and decreased swallowing function. Therefore, this study was to provide Home Visit Oral Health Education (HVOE) to some community care participants, and carried out to determine the effect of dry mouth in the elderly.

Methods: The study subjects were set as 20 in the experimental group and 20 in the control group. The intervention group was given oral muscle function strengthening exercise through video theory education and practice for 6 weeks. The researcher visited the subject's home and performed HVOE for about 50 minutes once a week for a total of 6 weeks.

Results: Homogeneity in all variables except for the general characteristics and non-stimulatory salivary flow of the intervention group and control group were confirmed $(\mathrm{p}>0.05)$. The 6-week HVOE had a significant effect on the change in non-stimulatory salivary flow $(\mathrm{p}<0.001)$. The value of controlling non-stimulatory saliva estimated before the education increased significantly more in the intervention group ( $7.19 \pm 0.19)$ compared to that of the control group $(5.04 \pm 0.19)(\mathrm{p}<0.001)$. A significant interaction between the intervention group and the time point was found for subjective mouth dryness $(\mathrm{F}=30.265, \mathrm{p}<0.001)$. The intervention group showed a more significant decrease than the control group with a decrease from $M=30.75$ to $M=18.50$ ( $p<0.001$ ).

Conclusions: The 6-week HVOE was found to have significant effects on the elderly who participated in the integrated community health care, showing decreased mouth dryness and non-stimulated saliva flow rate. Therefore, HVOE program for the elderly by local communities is highly expected to greatly contribute to improving and promoting oral and physical health.

Keywords: Community health care, Elderly, Mouth dryness, Oral health education

Copyright (C) 2021. Korean Academy of Preventive Dentistry. All rights reserved.

This is an Open Access article distributed under the terms of the Creative Commons Attribution Non-Commercial License (http://creativecommons.org/licenses/ by-nc/4.0) which permits unrestricted non-commercial use, distribution, and reproduction in any medium, provided the original work is properly cited. 